\title{
Spin Transfer Torque in Ferromagnet-Normal Metal-Antiferromagnet Junctions
}

\author{
Hyun-Woo Lee ${ }^{1 *}$ and Hyunsoo Yang ${ }^{2}$ \\ ${ }^{1}$ PCTP and Department of Physics, Pohang University of Science and Technology, Pohang, Kyungbuk 790-784, Korea \\ ${ }^{2}$ Department of Electrical and Computer Engineering, National University of Singapore, 117576, Singapore
}

(Received 28 April 2011, Received in final form 20 May 2011, Accepted 25 May 2011)

\begin{abstract}
This study investigated theoretically the properties of the spin transfer torque acting on a ferromagnet in a ferromagnet-normal metal-antiferromagnet junction. Earlier work showed that the angular dependence of the spin transfer torque can be a wavy-type if the junction satisfies a special symmetry. This paper reports a simple model analysis that allows a derivation of the wavy angular dependence without taking advantage of the symmetry. This result suggests that the wavy angular dependence can appear even when the symmetry is broken. As an illustration, the angular dependence was calculated as a function of the degree of the compensation at the normal metal-antiferromagnet interface. The implications of the result for the current-induced magnetization precession are discussed.
\end{abstract}

Keywords: spin transfer torque, antiferromagnet, microwave generation, current-induced magnetization precession

\section{Introduction}

The exchange of the spin angular momentum between the conduction electrons and local magnetization generates the spin transfer torque (STT) [1-3]. The STT provides an efficient means of controlling magnetic states of nanostructures and has potential applications toward memory, logic and microwave devices [4-6]. The early stage works [7-16] on the STT focused on the effect of the STT on the F-N-F structure, where "F" and "N" stand for a ferromagnet and normal metal, respectively. The current-induced magnetization switching (CIMS) [11, 13] and current-induced magnetization precession (CIMP) $[10,12-15]$ have been demonstrated. Recent studies [1726] also addressed the effect of the STT in the F-I-F magnetic tunneling junction (MTJ), where "I" stands for an insulator. In particular, the MgO-based MTJ [19-26] has received special attention owing to its high magnetoresistance [27-30]. Again both the CIMS [21, 23, 26] and CIMP [22] have been demonstrated. Various details of STT physics are under active investigation [31-36] and a range of extensions are being explored [37-40].

Although both the F-N-F and F-I-F structures share the phenomena of the CIMS and the CIMP, the detailed properties differ considerably because depending on the struc-

\footnotetext{
*Corresponding author: Tel: +82-54-279-2092
}

Fax: +82-54-279-3099, e-mail: hwl@postech.ac.kr tures, the STT has different current and angular dependences. In the F-N-F structure, the STT $\mathbf{T}$ acting on the free layer magnetization $\mathbf{M}$ is described [3] by the following

$$
\mathbf{T}=I_{e} g_{\mathrm{FNF}}(\theta) \mathbf{M} \times(\mathbf{M} \times \mathbf{P}),
$$

where $\mathbf{P}$ stands for the pinned layer magnetization, $I_{e}$ is the current flowing through the F-N-F structure, and $\theta$ is the angle between $\mathbf{M}$ and $\mathbf{P}$. Here $g_{\mathrm{FNF}}(\theta)$ is given approximately [3] by $q /(A+B \cos \theta)$, where $q, A, B$ are constants. On the other hand, in the F-I-F structure, the STT $\mathbf{T}$ acting on the free layer magnetization $\mathbf{M}$ is described $[17,18]$ as follows:

$$
\mathbf{T}=f_{\text {in-plane }}(V) g_{\text {in-plane }}(\theta) \mathbf{M} \times(\mathbf{M} \times \mathbf{P})+f_{\text {perp }}(V) g_{\text {gerp }}(\theta) \mathbf{M} \times \mathbf{P},(2)
$$

where, in contrast to Eq. (1), the so-called perpendicular STT in the direction of $\mathbf{M} \times \mathbf{P}$ is also present and comparable in magnitude to the in-plane STT in the direction of $\mathbf{M} \times(\mathbf{M} \times \mathbf{P})$. Both $g_{\text {in-plane }}(\theta)$ and $g_{\text {perp }}(\theta)$ are believed to be $1[17,18,20]$, whereas the voltage dependences $f_{\text {in-plane }}(V)$ and $f_{\text {perp }}(V)$ of the in-plane and perpendicular STTs are less clear. In particular, there is ongoing debate [19-21, 23-26] regarding the voltage dependence of the perpendicular STT.

This study examined the STT in the F-N-AF structure (Fig. 1), where AF stands for the antiferromagnet. It was predicted theoretically [41, 42, 45-47] that the STT effects also arise in magnetic nanostructures containing an $\mathrm{AF}$ 


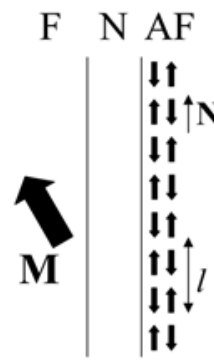

(a)

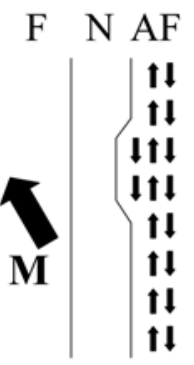

(b)

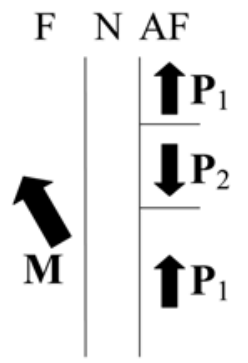

(c)
Fig. 1. Schematic diagram of the F-N-AF structure. (a) The AF layer is completely compensated in the sense that the vector sum of the magnetization at the N-AF interface is zero. (b) The AF layer is partially compensated because the vector sum of the magnetization at the N-AF interface is not zero. (c) A model of the F-N-AF structure, where the AF layer is modeled by ferromagnetic regions with alternating magnetizations $\mathbf{P}_{1}$ and $\mathbf{P}_{2}$.

layer, and some effects have been indeed observed experimentally $[43,44]$. A theoretical study [46] reported that when the magnetic configuration at the N-AF interface is completely compensated and thus possesses a certain special symmetry, the angular dependence of the STT acting on the $\mathrm{F}$ layer is a wavy-type (Fig. 2). Based on simple model analysis, the present study suggests that the angular dependence of the STT in the F-N-AF structure can still be a wavy-type for more general types of magnetic ordering at the N-AF interface, which does not possess the special symmetry used in Ref. [46]. In this sense, the present study generalizes Ref. [46]. Section 2 presents the theoretical analysis, which gives insights into properties of the STT in the F-N-AF structure. Section 3 discusses the implications of the results. As discussed in Sec. 3, the wavy STT may have considerable merit over the conventional angular dependences in the F-N-F structure or the F-I-F structure because it allows the CIMP without an external magnetic field. Section 4 concludes this paper.

\section{Angular Dependence of F-N-AF Structure}

This section addresses the angular dependence of the STT acting on the F layer in the F-N-AF structure. The F$\mathrm{N}-\mathrm{AF}$ structure with a completely compensated AF layer was first considered [Fig. 1(a)]. Reference [46] reported that the STT T acting on the F layer in the F-N-AF structure can be given by Eq. (1) with $\mathbf{P}$ replaced by the Neel vector $\mathbf{N}$ [Fig. 1(a)] and $g_{\mathrm{FNF}}(\theta)$ replaced by $g_{\mathrm{FNAF}}(\theta)$,

$$
g_{\mathrm{FNAF}}(\theta)=\frac{\tilde{g}_{\mathrm{FNAF}}(\theta)}{\sin \theta},
$$

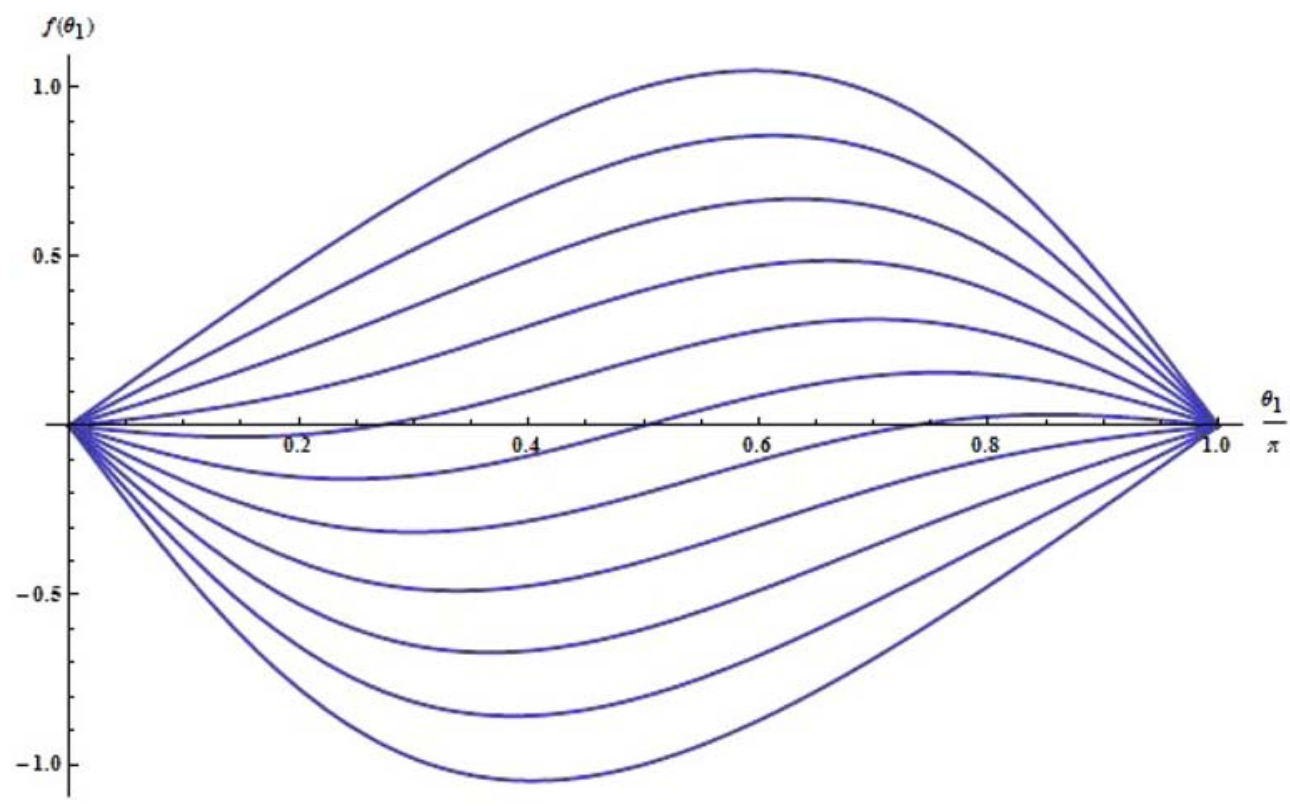

Fig. 2. The angular dependence of the STT acting on the F layer in the F-N-AF structure. Each curve shows the dependence for different degrees of compensation in the AF layer. The weighting factor $w_{1}$, which parameterizes the degree of compensation in the AF layer, varies from 1.0 (top curve) to 0.0 (bottom curve) in steps of 0.1 . Note that the graphs for $w_{1}=0.4,0.5,0.6$ exhibit a sign change. Therefore, in a certain range of $w_{1}$ near the complete compensation point $\left(w_{1}=0.5\right)$, the dependence is of the wavy-type. For simplicity, the graphs are provided for only one polarity of the current bias. The sign of the STT should be reversed when the current bias direction is reversed. In this figure, the approximation [3] $g_{\mathrm{FNF}}(\theta)=q /(A+B \cos \theta)$ is used with $q=1, A=1$, and $B / A=0.4$. The horizontal axis represents $\theta_{1} / \pi$ and the vertical axis represents $f\left(\theta_{1}\right) \equiv \sin \theta_{1}\left[w_{1} g_{\mathrm{FNF}}\left(\theta_{1}\right)-w_{2} g_{\mathrm{FNF}}\left(\pi-\theta_{1}\right)\right]$. 
has the series expansion form,

$$
\tilde{g}_{\mathrm{FNAF}}(\theta)=\sum_{n=1}^{\infty} \tilde{g}_{n} \sin (2 n \theta) \text {. }
$$

Here $\tilde{g}_{n}$ is the expansion coefficient. A striking prediction of Eq. (4) is that $g_{\mathrm{FNAF}}(\theta)$ vanishes at $\theta=\pi / 2$. This is in contrast to the results obtained for the F-N-F or F-I-F structures, where the STT vanishes only at two angles, $\theta=0$ and $\pi$. Therefore, the magnitude of the STT in the F-N-AF structure follows a wavy behavior in Fig. 2 with sign reversal occurring at $\theta=\pi / 2$. Here the positive and negative signs suggest that the direction of the STT is parallel and anti-parallel to $\mathbf{M} \times(\mathbf{M} \times \mathbf{N})$, respectively.

In Ref. [46], Eq. (4) was derived from the symmetry of the completely compensated $\mathrm{AF}$ without a disorder; the system remains invariant when the local magnetization in the AF layer is reversed and subsequently translated laterally by half of the lattice constant $l$ of the Neel ordering [Fig. 1(a)]. Although the derivation is elegant, it does not allow a generalization to a more generic AF, e.g. partially compensated AF, because the symmetry does not exist in general situations. An alternative method, which does not take advantage of the symmetry, is needed to generalize this result. For accurate and quantitative predictions of the STT in the F-N-AF structure, extensive first principles calculations are probably needed. This paper is confined instead to a much simpler model analysis. Although this analysis is not reliable on a quantitative level, it still captures the qualitative behavior of the STT correctly. In this simple analysis, a general AF layer [Fig. 1(b)] was modeled as an alternating sequence of $\mathrm{F}$ regions with the magnetization direction of each $\mathrm{F}$ region alternating in sequence [Fig. 1(c)]. Let $\mathbf{P}_{1}$ and $\mathbf{P}_{2}\left(\mathbf{P}_{2}=-\mathbf{P}_{1}\right)$ denote the alternating magnetization directions of the $\mathrm{F}$ regions in the AF layer. The core assumption of this simple analysis was to assume that the total STT $\mathbf{T}$ can be expressed as the sum of two contributions, one representing the STT for the FNF structure with the pinned layer magnetization $\mathbf{P}_{1}$ and the other representing the STT for the FNF structure with the pinned layer magnetization $\mathbf{P}_{2}$. Depending on the degree of compensation in the $\mathrm{AF}$ layer, the relative weighting of $\mathbf{P}_{1}$ and $\mathbf{P}_{2}$ will vary. For a general AF layer, the weighting consideration leads to

$$
\mathbf{T}=w_{1} I_{e} g_{\mathrm{FNF}}\left(\theta_{1}\right) \mathbf{M} \times\left(\mathbf{M} \times \mathbf{P}_{1}\right)+w_{2} I_{e} g_{\mathrm{FNF}}\left(\theta_{2}\right) \mathbf{M} \times\left(\mathbf{M} \times \mathbf{P}_{2}\right),
$$

for the STT $\mathbf{T}$ acting on the $\mathrm{F}$ layer in the F-N-AF structure. Here the weighting factors $w_{1}$ and $w_{2}\left(w_{1}+w_{2}\right.$ $=1$ ) parameterize the relative weightings of the $\mathbf{P}_{1^{-}}$and $\mathbf{P}_{2}$-magnetized areas at the N-AF interface. The angles $\theta_{1}$ and $\theta_{2}$ denote the angles between $\mathbf{M}$ and $\mathbf{P}_{1}$ and between $\mathbf{M}$ and $\mathbf{P}_{2}$, respectively. Although Eq. (5) is not exact, it still captures the essential qualitative properties. For example, in the limiting case, $w_{1}=1, w_{2}=0$, the result should reduce to that of the F-N-F structure, which is indeed the case. In the limiting case, $w_{1}=w_{2}=1 / 2$, Eq. (5) should reproduce the result in Ref. [46] for the F-NAF structure with a completely compensated AF layer. To confirm this, one utilizes the relations, $\mathbf{P}_{2}=-\mathbf{P}_{1}$ and $\theta_{2}=\pi-\theta_{1}$, to obtain

$$
\mathbf{T}=I_{e} \frac{g_{\mathrm{FNF}}\left(\theta_{1}\right)-g_{\mathrm{FNF}}\left(\pi-\theta_{1}\right)}{2} \mathbf{M} \times\left(\mathbf{M} \times \mathbf{P}_{1}\right) .
$$

Note that $\left[g_{\mathrm{FNF}}\left(\theta_{1}\right)-g_{\mathrm{FNF}}\left(\pi-\theta_{1}\right)\right] / 2$ is the counterpart of $g_{\mathrm{FNAF}}(\theta)$ in Eq. (3). It is trivial to verify that $\left[g_{\mathrm{FNF}}\left(\theta_{1}\right)\right.$ $\left.-g_{\mathrm{FNF}}\left(\pi-\theta_{1}\right)\right] / 2$ satisfies the following three properties; (i) $\left[g_{\mathrm{FNF}}\left(\theta_{1}\right)-g_{\mathrm{FNF}}\left(\pi-\theta_{1}\right)\right] / 2$ vanishes when $\theta_{2}=\theta_{1}=\pi / 2$, (ii) $\sin \theta_{1}\left[g_{\mathrm{FNF}}\left(\theta_{1}\right)-g_{\mathrm{FNF}}\left(\pi-\theta_{1}\right)\right] / 2$ is an odd function of $\theta_{1}$, and (iii) $\sin \theta_{1}\left[g_{\mathrm{FNF}}\left(\theta_{1}\right)-g_{\mathrm{FNF}}\left(\pi-\theta_{1}\right)\right] / 2$ is a periodic function of $\theta_{1}$ with the period $\pi$. Properties (ii) and (iii) can be confirmed by utilizing the properties that $g_{\mathrm{FNF}}(\theta)$ is an even function of $\theta$ and periodic with a period $2 \pi$. The properties (ii) and (iii) guarantee that $\sin \theta_{1}\left[g_{\mathrm{FNF}}\left(\theta_{1}\right)-\right.$ $\left.g_{\mathrm{FNF}}\left(\pi-\theta_{1}\right)\right] / 2$ can be expanded as a sum of $\sin 2 n \theta_{1}$, just like $\tilde{g}_{\mathrm{FNAF}}(\theta)$ in Eq. (4). This demonstrates that the simple model captures the core properties of both the F$\mathrm{N}-\mathrm{F}$ structure and F-N-AF structure with a completely compensated AF layer.

For a general AF layer, the degree of the compensation varies, suggesting that $w_{1}$ can vary from 0 (complete uncompensation) to 1 (also complete uncompensation) through $1 / 2$ (complete compensation). Figure 2 shows the magnitude of $\mathbf{T}$ as a function of the angle $\theta_{1}$ for selected values of $w_{1}$. Again the positive and negative signs suggest that $\mathbf{T}$ is parallel and antiparallel to $\mathbf{M} \times\left(\mathbf{M} \times \mathbf{P}_{1}\right)$. Note that in a finite range of $w_{1}$ near $1 / 2$, the angular dependence exhibits sign reversal and the angular dependence becomes a wavy-type. Compared to Ref. [46], where the wavy angular dependence is derived from the special symmetry that exists only at the complete compensation point $\left(w_{1}=1 / 2\right)$, this simple model analysis shows that the wavy angular dependence can be obtained without using the symmetry. This suggests that the wavy angular dependence should be robust with respect to various types of irregularities, such as atomic disorders in the N-AF interface, magnetic disorders within the AF layer provided they are not too strong. This is the main result of this paper.

\section{Discussion}

The property of the CIMP depends critically on the angular dependence of the STT. One of the most impor- 
tant technical goals in view of microwave generation from magnetic nanostructures is to achieve a CIMP without an external magnetic field. This is believed to be difficult for conventional F-N-F and F-I-F structures. One exceptional case is the reference [48], where the CIMP without an external magnetic field is realized in the F-N-F structure. This experimental result was attributed to the waviness of the STT. Reference [49] argued that the angular dependence of the STT in a strongly asymmetric F-N-F structure can become wavy due to the strong asymmetric spin accumulation pattern in such a structure. The F-N-F structure used in Ref. [48] indeed possesses strong asymmetry. Owing to its impact on the application potential, microwave oscillation generation without an external magnetic field has been pursued [50-54] in various ways. In this respect, the F-N-AF structure examined in this paper provides an alternative way to realize the wavy STT. The presence of an alternative method to realize the wavy STT enhances the engineering degrees of freedom in view of device applications.

\section{Conclusion}

The STT in the F-N-AF structure was examined. The STT in this structure can have a wavy angular dependence and the degree of the waviness depends on the degree of the compensation in the AF layer. The wavy angular dependence of the STT allows the current-induced magnetization precession even in the absence of an external magnetic field. Therefore, this result might be useful for the device designs of microwave generator based on magnetic nanostructures. Experimental efforts to test the predicted wavy STT will be needed. A quantitative comparison between the experimental results and the simplified model in this paper may have impact on possible device applications. Furthermore, it may reveal limitations of the simplified model used in this paper and suggest future directions towards more reliable quantitative theories of the STT in the F-N-AF structure.

\section{Acknowledgement}

This study was supported financially by the Korea Research Foundation Grant (KRF-2009-013-C00019). HY was supported by the Singapore National Research Foundation under CRP Award No. NRF-CRP 4-2008-06. HWL acknowledges the hospitality of the National University of Singapore, where this work was initiated. HWL also acknowledges Soo-Yong Lee for his technical assistance in manuscript preparation.

\section{References}

[1] L. Berger, Phys. Rev. B 54, 9353 (1996).

[2] J. C. Slonczewski, J. Magn. Magn. Mater. 159, L1 (1996).

[3] D. C. Ralph and M. D. Stiles, J. Magn. Magn. Mater. 320, 1190 (2008).

[4] H. Koo, C. Krafft, and R. D. Gomez, Appl. Phys. Lett. 81, 862 (2002).

[5] S. S. P. Parkin, U. S. Patent No. 6,834,005 (2004).

[6] D. A. Allwood, G. Xiong, C. C. Faulkner, D. Atkinson, D. Petit, and R. P. Cowburn, Science 309, 1688 (2005).

[7] J. Z. Sun, Phys. Rev. B 62, 570 (2000).

[8] J. C. Slonczewski, J. Magn. Magn. Mater. 247, 324 (2002).

[9] S. Zhang, P. M. Levy, and A. Fert, Phys. Rev. Lett. 88, 236601 (2002).

[10] M. Tsoi, A. G. M. Jansen, J. Bass, W.-C. Chiang, M. Seck, V. Tsoi, and P. Wyder, Phys. Rev. Lett. 80, 4281 (1998).

[11] E. B. Myers, D. C. Ralph, J. A. Katine, R. N. Louie, and R. A. Buhrmann, Science 285, 867 (1999).

[12] M. Tsoi, A. G. M. Jansen, J. Bass, W.-C. Chiang, V. Tsoi, and P. Wyder, Nature 406, 46 (2000).

[13] J. A. Katine, F. J. Albert, R. A. Buhrman, E. B. Myers, and D. C. Ralph, Phys. Rev. Lett. 84, 3149 (2000).

[14] S. I. Kiselev, J. C. Sankey, I. N. Krivorotov, N. C. Emley, R. J. Schoelkopf, R. A. Buhrman, and D. C. Ralph, Nature 425, 380 (2003).

[15] I. N. Krivorotov, N. C. Emley, J. C. Sankey, S. I. Kiselev, D. C. Ralph, and R. A. Buhrman, Science 307, 228 (2005).

[16] K.-J. Lee, A. Deac, O. Redon, J.-P. Nozieres, and B. Dieny, Nature Mater. 3, 877 (2004).

[17] J. C. Slonczewski, Phys. Rev. B 71, 024411 (2005).

[18] I. Theodonis, N. Kioussis, A. Kalitsov, M. Chshiev, and W. H. Butler, Phys. Rev. Lett. 97, 237205 (2006).

[19] H. Kubota, A. Fukushima, K. Yakushiji, T. Nagahama, S. Yuasa, K. Ando, H. Maehara, Y. Nagamine, K. Tsunekawa, D. D. Djayaprawira, N. Watanabe, and Y. Suzuki, Nature Phys. 4, 37 (2008).

[20] J. C. Sankey, Y.-T. Cui, J. Z. Sun, J. C. Slonczewski, R. A. Buhrman, and D. C. Ralph, Nature Phys. 4, 67 (2008).

[21] Z. Li, S. Zhang, Z. Diao, Y. Ding, X. Tang, D. M. Apalkov, Z. Yang, K. Kawabata, and Y. Huai, Phys. Rev. Lett. 100, 246602 (2008).

[22] A. M. Deac, A. Fukushima, H. Kubota, H. Maehara, Y. Suzuki, S. Yuasa, Y. Nagamine, K. Tsunekawa, D. D. Djayaprawira, and N. Watanabe, Nature Phys. 4, 803 (2008).

[23] S.-C. Oh, S.-Y. Park, A. Manchon, M. Chshiev, J.-H. Han, H.-W. Lee, J.-E. Lee, K.-T. Nam, Y. Jo, Y.-C. Kong, B. Dieny, and K.-J. Lee, Nature Phys. 5, 898 (2009).

[24] M. H. Jung, S. Park, C.-Y. You, and S. Yuasa, Phys. Rev. B 81, 134419 (2010).

[25] O. G. Heinonen, S. W. Stokes, and J. Y. Yi, Phys. Rev. Lett. 105, 066602 (2010).

[26] S.-Y. Park, J.-H. Han, S.-C. Oh, J.-E. Lee, K.-T. Nam, H.-W. Lee, Y. Jo, and K.-J. Lee, J. Phys. D: Appl. Phys. 
44, 064008 (2011).

[27] W. H. Butler, X.-G. Zhang, T. C. Schulthess, and J. M. MacLaren, Phys. Rev. B 63, 054416 (2001).

[28] J. Mathon and A. Umerski, Phys. Rev. B 63, 220403(R) (2001).

[29] S. S. P. Parkin, C. Kaiser, A. Panchula, P. M. Rice, B. Hughes, M. Masant, and S.-H. Yang, Nature Mater. 3, 862 (2004).

[30] S. Yuasa, T. Nagahama, A. Fukushima, Y. Suzuki, and K. Ando, Nature Mater. 3, 868 (2004).

[31] W. J. Kim, T. D. Lee, J. E. Lee, S. C. Oh, K. H. Shin, H. J. Suh, and K.-J. Lee, Appl. Phys. Lett. 90, 212504 (2007).

[32] J. Ryu and H.-W. Lee, J. Appl. Phys. 105, 093929 (2009).

[33] W. J. Kim, K.-J. Lee, and T. D. Lee, J. Magnetics 14, 104 (2009).

[34] S. W. Lee and K.-J. Lee, J. Kor. Phys. Soc. 55, 1501 (2009).

[35] C.-Y. You, S.-S. Ha, and H.-W. Lee, J. Magn. Magn. Mater. 321, 3589 (2009).

[36] K.-W. Kim and H.-W. Lee, Phys. Rev. B 82, 134431 (2010).

[37] C. Y. You, Appl. Phys. Lett. 92, 152507 (2008).

[38] S.-W. Jung, W. Kim, T.-D. Lee, K.-J. Lee, and H.-W. Lee, Appl. Phys. Lett. 92, 202508 (2008).

[39] S. M. Seo, H. Yang, T. Ono, and K. J. Lee, Phys. Rev. Lett. 102, 147202 (2009).

[40] A. Flandimas, K. J. Lee, and K.-H. Shin, J. Magnetics 14, 90 (2009).

[41] A. S. Nunez, R. A. Duine, P. Haney, and A. H. MacDonald, Phys. Rev. B 73, 214426 (2006).
[42] R. A. Duine, P. M. Haney, A. S. Nunez, and A. H. MacDonald, Phys. Rev. B 75, 014433 (2007).

[43] Z. Wei, A. Sharma, A. S. Nunez, P. M. Haney, R. A. Duine, J. Bass, A. H. MacDonald, and M. Tsoi, Phys. Rev. Lett. 98, 116603 (2007).

[44] S. Urazhdin and N. Anthony, Phys. Rev. Lett. 99, 046602 (2007).

[45] Y. Xu, S. Wang, and K. Xia, Phys. Rev. Lett. 100, 226602 (2008).

[46] P. M. Haney and A. H. MacDonald, Phys. Rev. Lett. 100, 196801 (2008).

[47] E. V. Gomonai and V. M. Loktev, Low Temp. Phys. 34, 198 (2008).

[48] O. Boulle, V. Cros, J. Grollier, L. G. Pereira, C. Deranlot, F. Petroff, G. Faini, J. Barnas, and A. Fert, Nature Phys. 3, 492 (2007).

[49] J. Barnas, A. Fert, M. Gmitra, I. Weymann, and V. K. Dugaev, Phys. Rev. B 72, 024426 (2005).

[50] K.-J. Lee, O. Redon, and B. Dieny, Appl. Phys. Lett. 86, 022505 (2005).

[51] D. Houssameddine, U. Ebels, B. Delaet, B. Rodmacq, I. Firastrau, F. Ponthenier, M. Brunet, C. Thirion, J. P. Michel, L. Prejbeanu-Buda, M. C. Cyrille, O. Redon, and B. Dieny, Nature Mater. 6, 447 (2007).

[52] V. S. Pribiag, I. N. Krivorotov, G. D. Fuchs, P. M. Baraganca, O. Zatay, J. C. Sankey, D. C. Ralph, and R. A. Buhrman, Nature Phys. 3, 498 (2007).

[53] C.-Y. You, J. Magnetics 14, 168 (2009).

[54] S.-W. Lee and K.-J. Lee, IEEE Trans. Magn. 46, 2349 (2010) 